\title{
COSMOLOGICAL IMPLICATIONS (DISCUSSION)
}

Discussion of the paper presented by CONDON (p. 535)

Stocke: Since real limits are surface brightness limits not flux, please comment on the possibility of sources missed because they have very low surface brightness.

Condon: Magellanic irregular galaxies have radio surface brightness comparable with the rms brightness fluctuation $(\approx 0.1 \mathrm{~K}$ at $1.4 \mathrm{GHz})$ of the radio source background, so they cannot be detected reliably even by the most sensitive radio telescope. The Wrobel \& Heeschen (1991) sample of E/SO AGNs appears to be brightness limited by the VLA observations with $5^{\prime \prime}$ resolution. This limitation can be corrected by the NVSS, which reaches to within an order-of-magnitude of the confusion limit, and Bill Cotton's preliminary results on the UGC galaxies (this volume) reveals a significant new population of low-brightness radio sources in elliptical galaxies.

Ekers: Your simple model to fit the source counts implies that the "normal galaxy" population has the same strong evolution seen in the powerful radio source population.

Condon: That's right. Even though the energy sources (stars and AGN) are quite different, the amount of evolution is about the same. This suggests a common evolutionary mechanism. For example, galaxy collisions are known to trigger starbursts, and they may also increase the fueling rate of central "monsters".

Lari: I wonder if the different estimates of radio luminosity function don't reflect that different type of galaxies have different (optical?) luminosity function. Different luminosity function would reflect the local inhomogeneity.

Condon: If different types of Galaxies (eg., spirals and ellipticals) all had the same radio/optical ratio $R$, then their radio luminosity functions would mimic their optical luminosity functions, just as the radio and FIR luminosity functions of "normal" galaxies are alike. In fact, the R-distributions of spirals and ellipticals are quite different. This broad 
range of radio properties is more important than the optical luminosity function differences in accounting for the differing radio luminosity functions of different galaxy types.

Lari: Counts are not always corrected for statistical bias.

Condon: I don't understand; does "counts" refer to source counts (n(s)) or numbers of galaxies in luminosity function bins? What statistical bias?

Discussion of the paper presented by WALL (p. 547)

Padovani: Your "beamed" objects include only the flat-spectrum sources. There are various indicators that steep-spectrum radio quasars are also beamed, although at somewhat larger angles. Did you try to include them in your calculations?

Wall: Our "beamed" objects were in fact drawn from the steep-spectrum luminosity function, using the high-power (evolving) end only. As yet we have not indulged in any refinements of the type you mention; but further work will incorporate them.

Discussion of the paper presented by FOMALONT (p. 555)

de Bruyn: The count convergence at $100 \mathrm{nJy}$ depends on the integrated sky temperature value of $0.02 \mathrm{~K}$ (excluding MWB). What is the error on this number and how would you determine it?

Fomalont: The sky brightness temperature has been measured by COBE above $19 \mathrm{GHz}$ and from the ground above $5 \mathrm{GHz}$. All measurements are consistent with a constant $\mathrm{CMB}$ of $2.7 \mathrm{~K}$ at all these frequencies to an error of no more than $0.02 \mathrm{~K}$. Hence, any residual sky brightness caused by radio sources at $8.1 \mathrm{GHz}$ must be less than about $0.02 \mathrm{~K}$.

Discussion of the paper presented by HAMMER (p. 559)

Becker: Have you calculated the chance coincidence rate between radio and optical sources?

Hammer: Not exactly. But the coincidence between radio and optical was much better than $1^{\prime \prime}$ for all sources but two. 
Wilson: You said that $25 \%$ of the radio sources are identified with low redshift, low luminosity AGN's and referred to them as Seyfert 2 or Seyfert 1 . You also said these objects have inverted radio spectra. Such radio spectra are extremely unusual among normal Seyfert galaxies. Do you have any suggestions about this difference in the radio spectra?

Hammer: These objects have also other differences with "normal" Seyferts. - their radio power might be higher $\left(P \gtrsim 10^{23} \mathrm{~W} / \mathrm{Hz}\right)$

- their emission line ratios $\left(\mathrm{OIII} / \mathrm{H}_{\beta}, \mathrm{SII} / \mathrm{H}_{\alpha}\right)$ are often intermediate between LINERS and Seyfert

- their optical luminosities are rather small (substantially smaller than $\left.L^{*}\right)$.

Wall: A very large proportion of radio sources in the $10-100 \mathrm{mJy}$ range turn out to be red ellipticals which obey a tight Hubble relation and which have no evidence of emission lines. These are 'proper' double-structure 'AGNs', real radio sources. It may be that your faint passive ellipticals are from the same population.

Discussion of the paper presented by $\underline{\operatorname{SHAVER}}$ (p. 561)

Jauncey: I am concerned that your selection of "no $B$-band" is based on spectra of optically selected QSOs, which were chosen on the basis of optical colours, in particular for having no $B$-band emission. So your selection for spectroscopy of "no $B$-band" is not necessarily a property of the quasars, but of the optical survey. It is known that several of the radio QSOs show emission shortward of the $912 \AA$ limit at the emission line redshift.

Shaver: This criterion is based on a property of the intervening medium, not of the QSOs themselves. At $z>4-5$ the increasing density of intervening Lyman-limit absorbers at high redshift blocks out the flux from the QSO right up to the Lyman limit in the QSO rest frame (5470 $\AA$ at $z=5$ ). Spectra of the highest-redshift radio-selected quasars do indeed exhibit this phenomenon (cf. Hook et al. 1995, MNRAS 271, L63; Shaver, Wall, Kellermann 1995, MNRAS in press).

Meisenheimer: How did you account for the radio luminosity function? If it is steep you will only see the most luminous (and rare) objects at high redshift. This has to be folded into your $\Phi(z)$ diagram.

Shaver: We are just concerned about the space density above a given luminosity. The computed $\Phi(z)$ corresponds to all luminosities above 
the minimum which could be detected at the highest redshift considered, $z=7$; the space density of quasars above that luminosity decreases at high redshift.

Bicknell: How do you know that high redshift QSOs are not free-free absorbed by surrounding ionized gas?

Shaver: Our main conclusion concerns intervening absorption along the line of sight; absorption by gas and dust intrinsic to the QSO does not affect that conclusion. We are talking about a particular observed phenomenon i.e. quasars like those seen at $z \sim 1-2$, and the space density of such objects decreases at high redshift. Of course there must be precursors of "normal" quasars (and galaxies) at still higher redshifts, at least in the form of mass concentrations, but that is a different issue. And even if there are "hidden" quasars enshrouded in dust and ionized gas, if quasars are young objects we would expect such "hidden" quasars to exist at all redshifts, so the redshift turnover would be unaffected. Any such objects may be found by conducting searches in the $\mathrm{mm} / \mathrm{IR}$ or X-ray bands.

Wright: Could you repeat the argument as to why the turnover in space density applies to all QSOs (rather than just radio-loud QSOs)?

Shaver: Potentially the most compelling argument concerns the UV background intensity measured in situ as a function of redshift using the "proximity effect" observed in the statistics of the Ly $\alpha$ forest in QSO spectra (see fig. 2 Bechtold, 1995 QSO Absorption Lines, ed. G. Meylan, ESO Astrophysics Symposia, Springer, p. 299). The UV field also seems to exhibit a pronounced maximum as a function of redshift. This coincides roughly with the redshift where the QSO space density peaks, consistent with the UV field having been produced by the QSOs (and other AGN), and suggestive of a universal turnover.

Vermeulen: What is known about the properties (redshifts, luminosities) of the radio galaxies in your sample?

Shaver: We have just begun a study of these optically-faint, flat-spectrum radio galaxies - it is too early to summarize their properties at the moment.

Discussion of the paper presented by SINGAL (p. 563)

Gurvits: Similar studies but on the $10^{3}$ times smaller angular scale (i.e. VLBI) indicate a clear difference in the behaviour of $(\theta-z)$ diagrams 
on the kpc and pc scales, certainly for quasars. Any comments on this difference?

Singal: If quasars differ from radio galaxies on pc or kpc scales then that is again an evidence against the simple unified scheme. However at those scales the size evolution may not be playing any part, at least not in the same way as for more extended structures. In fact the absence of a size evolution at that scale is assumed in interpreting the $(\theta-z)$ plots for determining $q_{0}$.

Leahy: As a member of Neeser et al., I'd like to make two comments:

1) We showed Oort et al suffered a major bias due to underestimating the size of distant FR1's through K-dimming.

2) You need complete redshift because the K- $z$ relation breaks down at $z \geq 2$. This is only available for $3 \mathrm{C}$ and our sample so far:- it is very important to complete the optical on big samples like Molonglo and B3.

Kapahi: I would like to make two comments:

1. One can make a fairly strong and simple argument (Kapahi 1989, A.J. 97, 1) for cosmological evolution of linear sizes that may not have been appreciated in the literature. FR II galaxies in the 3CR sample have a median linear size between $\sim 250$ and 300 kpc almost independent of redshift up to $z \sim 1$. The minimum angular size subtended by a $250 \mathrm{kpc}$ source (for $q_{0}=0.5$ ) at any redshift is $\sim 30^{\prime \prime}$. If linear sizes were independent of redshift and luminosity, median angular sizes $\left(\theta_{m}\right)$ in complete flux limited samples of radio galaxies can never be $<30^{\prime \prime}$. Whereas it is well known that $\theta_{m}$ approaches a value near $10^{\prime \prime}$ in complete surveys at flux levels between $\sim 1$ and $0.1 \mathrm{Jy}$ at $408 \mathrm{MHz}$. This can only be explained by invoking evolution in linear sizes as the deeper samples have higher median redshift. It is clearly important to check that models for the $\mathrm{z}$ and $\mathrm{P}$ dependence of linear sizes are consistent with the observed $\theta-S$ relation as well.

2. The fraction of compact steep-spectrum sources in source samples is a function of both survey frequency as well as the flux density limit. This can affect the comparison of median sizes in different samples and must be kept in mind while interpreting the comparisons.

Discussion of the paper presented by MEISENHEIMER (p. 571)

di Serego Alighieri: In your introduction you said that high redshift radio galaxies are particularly useful to study stellar systems in the early universe; you mentioned young stars to explain the blue colours. 
Can you say anything about the stellar content in the galaxies you have studied?

Meisenheimer: Not yet. So far we have concentrated on studying the emission line regions. A detailed investigation of the continuum colors (after subtracting the contribution of the emission lines) has still to be done.

Koekemoer: In your K-band data of $4 \mathrm{C} 41.17$, do you detect any companions which might be interacting - and if so, can you constrain the dynamics of Ly- $\alpha$ in the primary galaxy.

Meisenheimer: In fact, we found several neighbours on our K-band image of $4 \mathrm{C} \mathrm{41.17.} \mathrm{But} \mathrm{none} \mathrm{of} \mathrm{those} \mathrm{show} \mathrm{significant} \mathrm{Ly-} \alpha$ emission within about $\pm 1500 \mathrm{kms}^{-1}$ of the systemic redshift of $4 \mathrm{C} 41.17$. So we do not regard them as physical companions.

Bicknell: Do you find any correlation between excitation and velocity dispersion.

Meisenheimer: We can't tell since we only have Fabry- Pérot data for a single line (either [0II] or Ly- $\alpha$. For redshifts $z<0.8$ we could in principle determine an $[0 \mathrm{III}] /[0 \mathrm{II}]$ ratio, but we have not tried this up to now. 